\title{
The Movement for Global Mental Health
}

\author{
Vikram Patel, Pamela Y. Collins, John Copeland, Ritsuko Kakuma, Sylvester Katontoka, \\ Jagannath Lamichhane, Smita Naik and Sarah Skeen
}

\section{Summary}

The Movement for Global Mental Health is a coalition of individuals and institutions committed to collective actions that aim to close the treatment gap for people living with mental disorders worldwide, based on two fundamental principles: evidence on effective treatments and the human rights of people with mental disorders.

\section{Declaration of interest}

All authors are members of the Movement for Global Mental Health.
Vikram Patel (pictured) is a Wellcome Trust Senior Research Fellow in Clinical Science at the Centre for Global Mental Health at the London school of Hygiene and Tropical Medicine and the Sangath Centre in Goa, India. Pamela Y. Collins is the Director of the Office for Research on Disparities and Global Mental Health at the National Institute of Mental Health/ National Institutes of Health, USA. John Copeland is Emeritus Professor of Psychiatry at the University of Liverpool, UK, and immediate Past President, World Federation of Mental Health. Ritsuko Kakuma holds posts at the Health Systems Research and Consulting Unit, Centre for Addiction and Mental Health in Toronto, Canada, and the Department of Psychiatry and Mental Health at the University of Cape Town, South Africa. Sylvester Katontoka works for the Mental Health Users Network of Zambia. Jagannath Lamichhane is a journalist and member of the Nepal Mental Health Foundation (NMHF) in Kathmandu. Smita Naik is at the Sangath Centre, Goa, India. Sarah Skeen works on the Mental Health and Poverty Project at the Department of Psychiatry and Mental Health, University of Cape Town, South Africa.

\section{Why the Movement for Global Mental Health?}

The Movement for Global Mental Health (the Movement, www.globalmentalhealth.org), launched on 10 October 2008, is a coalition of individuals and institutions committed to collective actions that aim to close the treatment gap for people living with mental disorders worldwide. The Movement focuses on those populations where the gaps are the largest: among people living in low- and middle-income (LAMI) countries. The Movement seeks to promote actions based on two fundamental principles: evidence on effective treatments and the human rights of people with mental disorders. The scientific foundation emerges from a wealth of evidence, synthesised in a series of landmark publications, notably the World Mental Health Report, ${ }^{1}$ the 2001 World Health Report, ${ }^{2}$ the Lancet series on global mental health $^{3}$ and the Public Library of Science (PloS) Medicine series on packages of care for mental disorders. ${ }^{4}$ On 7 October 2010, the World Health Organization (WHO) released is mhGAP intervention guidelines for scaling up services for mental, neurological and substance use disorders. ${ }^{5}$ The human rights foundation lies in established international instruments, notably the United Nations Convention on the Rights of Persons with Disabilities that calls for the full and effective participation and inclusion in society of people with disabilities, including people with mental disorders and with intellectual disabilities. The Movement takes its inspiration from HIV movements that have successfully mobilised significant global resources for the care of people living with HIV/AIDS in LAMI countries. Critical to the success of the global HIV campaign were its specific and clear calls to action, its grounding in the evidence of effective treatments and human rights, and its united front of people living with
HIV/AIDS, health practitioners, policy-makers and researchers sharing a common platform. The Movement seeks to emulate these characteristics, offering a platform where members from diverse backgrounds stand together as partners to promote the shared vision of closing the treatment gap.

\section{What is the Movement?}

The Movement was conceived as a follow-up strategy by the authors of the 2007 Lancet series, which culminated in a call to action to scale up services for people with mental disorders. ${ }^{3}$ About 60 people, identified through a snow-balling process, formed the founding advisory group of the Movement. This group brought together people affected by mental disorders and individuals representing human rights organisations, non-government organisations, professional societies, universities and global organisations (www.globalmentalhealth.org/articles.php?id=75). The group then collaborated through opportunistic meetings at conferences and by email to define the Charter, a set of core strategies and coordinators for these strategies. There was neither a formal secretariat nor a budget. Through volunteerism and collaboration, the Movement has gradually grown to a stage where members are able to share ideas, initiate activities and seek resources often in partnership with other members. Since its inception, the Movement has grown to a membership of 1304 individuals and 77 institutions (as of 31 July 2010). Over half the individual members $(n=786,60 \%)$ are women and nearly half $(40 \%)$ live in LAMI countries. Table 1 shows the breakdown of the membership by country category and stakeholder group. It is reassuring to note the significant representation of all constituencies, including people affected by mental disorders who comprise over a quarter of the membership. Figure 1 shows the characteristics of the institutional members; nearly half the institutions represent user groups or non-governmental organisations and just under a half $(n=36)$ are based in LAMI countries. Figure 2 shows the proportions of individual members by world region and the location of institutional members. Although the Movement has institutional members from 28 countries (10 high-income and 18 LAMI countries) and individual members from 103 countries (33 high-income and 70 LAMI countries), it is also evident that the membership is not representative of the global population. This is not surprising given the vastly different levels of development of the field of mental health in different countries. Another factor is language - thus, the relative scarcity of representation from non-English speaking countries is an important weakness that can and should be addressed. 


\begin{tabular}{|c|c|c|c|}
\hline & \multicolumn{2}{|r|}{$n(\%)$} & \multirow[b]{2}{*}{ Total } \\
\hline & High-income countries & Low- and middle-income countries & \\
\hline Person affected by mental disorder & $80(6)$ & $64(5)$ & $144(11)$ \\
\hline Family member of person affected by mental disorder & $126(10)$ & $80(6)$ & $206(16)$ \\
\hline Policy maker/advisor & $139(11)$ & $100(8)$ & 239 (18) \\
\hline Clinical practitioner or care provider & $382(29)$ & $312(24)$ & $694(53)$ \\
\hline Activist/advocate & $199(15)$ & 149 (11) & $348(27)$ \\
\hline Academic/researcher & 459 (35) & $302(23)$ & $761(58)$ \\
\hline Other & $35(3)$ & $28(2)$ & $63(5)$ \\
\hline
\end{tabular}

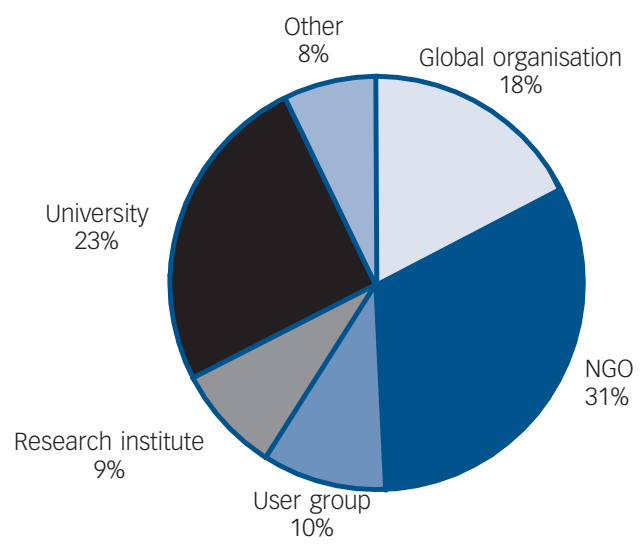

Fig. 1 Characteristics of the institutional members $(n=77)$. NGO, non-governmental organisation.

\section{What has the Movement been doing?}

The Movement does not seek to duplicate or compete with the actions of its members; it seeks to build a coalition whose diverse members share a common goal and who can use the Movement to support their own activities and strengthen the Movement simultaneously. In all countries, people affected by mental disorders experience discrimination at multiple levels in society, ${ }^{6}$ including within the healthcare system. Once affected by mental disorder, overcoming the layers of social barriers and discrimination is often a far greater challenge than overcoming the illness at the personal level. ${ }^{6}$ In spite of international instruments aimed at protecting the rights of people with mental disorders, this community remains among the most vulnerable and neglected groups in all societies. ${ }^{7}$ Against this backdrop, the struggle for social justice requires multiple actions, including an increase in availability of and access to a range of mental health services.

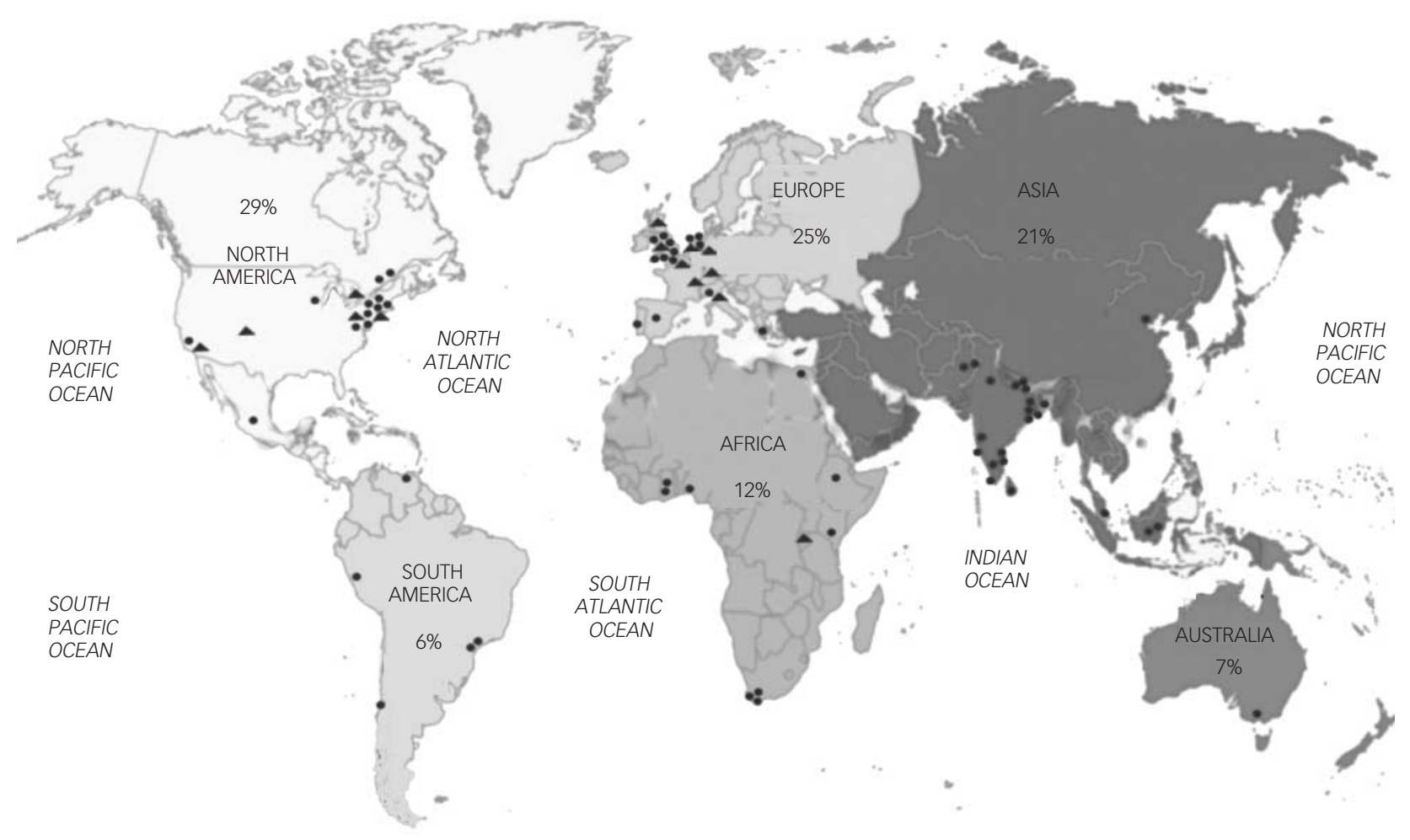

Fig. 2 Location of institutional members $(n=77)$ and regional distribution of individual members $(\%)(n=1304)$.

Global organisations are shown as triangles and are located at either the corresponding address of the organisation or its current president. Circles represent institutional partners. 
The demand for effective implementation of human rights instruments is an equally important goal.

The website (www.globalmentalhealth.org) plays a central role as a 'virtual headquarters' and houses resources such as packages of care, human rights stories and advocacy articles. Members can search databases and share resources by making online submissions. A monthly email newsletter alerts members about recent activities and updates, showcases new resources, and promotes engagement in Movement activities. In early 2009, the Movement's virtual reach was further expanded when a profile was added to the social networking site Facebook; this site currently has more than 800 members. Discussion boards were added in early 2010 to enhance communication between members. The first Global Mental Health Summit was held in Athens in September 2009, and provided an opportunity for members to meet face to face to share their experiences and initiate new collaborations. Keynote speakers included personalities who had spearheaded global HIV campaigns. Supporting capacity building in global mental health is one of the core aims of the Movement. A survey of capacity building initiatives was conducted in 2009 and the findings were used to develop a Capacity Building Atlas for Global Mental Health. This Atlas (www.globalmentalhealth.org/cb_atlas.html) provides information on 25 programmes that have been identified thus far and members can add new programmes using an online submission form. In October 2009, the Lancet, an institutional member of the Movement, initiated a plan for a new series of articles on global mental health to address themes that were not covered in the first series and to track progress made since then. Following a systematic process that began with a call for themes from the Movement membership, six articles have been commissioned and the series is expected to be launched at the second Global Mental Health Summit in Cape Town in October 2011.

\section{Where does the Movement go next?}

The Movement is the follow-up strategy to the call for scaling up services for people with mental disorders. ${ }^{3}$ A core philosophy of the Movement is to strengthen the platform on which all global mental health communities can build a partnership to take collective action for global mental health. More than evidence is needed to mobilise civil society on a mass scale. Those committed to the goals of the Movement need to show that we all agree on basic principles. Mental disorders are invisible in most societies and we need to make our cause visible to everyone by our numbers and by public action. We must insist on seeking recovery for all who have mental disorders and if rights are abused, then they must be shown to be abused, in all countries. To achieve this goal, the World Federation for Mental Health (WFMH), a global non-governmental organisation and a member of the Movement, is planning a major new advocacy initiative, the 'WFMH Great Push for Mental Health', in collaboration with the Movement, around the themes of 'Unity, Visibility, Rights, Recovery'.

Thus far, the activities of the Movement have grown organically with no explicit structure or plan. This is the very essence of social movements with a strong participatory philosophy. At the same time, the voluntary nature of activities dictates the pace and direction of the Movement's development.
Thus, the accomplishments so far are the result of initiatives by those committed to the cause, their willingness to find resources, and to volunteer their time. A major challenge for the Movement is to sustain it through these early years. The advisory board is currently considering a renewal of the leadership of the Movement to bring on board motivated individuals and establishing a rotating secretariat. For any social movement, flexibility and trust are vital to bind its diverse communities to a central goal. The Movement's members believe that a selfless moral struggle built on a partnership of professionals and civil society is essential to mutually strengthen all global mental health communities. The emergence of global mental health as a lively discipline of global health ${ }^{8}$ offers a timely opportunity for the Movement. The Movement aspires to an ever-widening membership and new activities to make mental health for all a reality - our strength ultimately lies in our numbers, our vision and our conviction. We invite you to join by visiting our website (www.globalmentalhealth.org).

\begin{abstract}
Vikram Patel, PhD, MRCPsych, Centre for Global Mental Health, London School of Hygiene \& Tropical Medicine, UK and the Sangath Centre, Goa, India; Pamela Y. Collins, MD MPH, National Institute of Mental Health/National Institutes of Health, USA J John Copeland, MD, SCD, Division of PSychiatry University of Liverpool, UK, Rits ; Ritsuko Kakuma, PhD, Health Systems Research and Consulting Unit, Centre for Addiction and Mental Health, Toronto, the Dalla Lana School of Public Health, University of Toronto, Canada and the Mental Health and Poverty Project, Department of Psychiatry and Mental Health, University of Cape Town, South Africa; Sylvester Katontoka, Diploma in Mental Health, Mental Health Users Network of Zambia; Jagannath Lamichhane, MA, Nepal Mental Health Foundation (NMHF), Kathmandu, Nepal: Smita Naik, BSc, Sangath Centre, Goa, India; Sarah Skeen, MPH, Mental Health and Poverty Project, Department of Psychiatry and Mental Health, University of Cape Town, South Africa
\end{abstract}

Correspondence: Vikram Patel, Sangath Centre, Alto-Porvorim, Goa 403521, India. Email: vikram.patel@Ishtm.ac.uk

First received 1 Apr 2010, final revision 22 Aug 2010, accepted 9 Sep 2010

\section{Funding}

V.P. is supported by a Wellcome Trust Senior Research Fellowship in Tropical Medicine. The views expressed in this article do not necessarily represent views of the National Institute of Mental Health or the US government.

\section{References}

1 Desjarlais R, Eisenberg L, Good B, Kleinman A. World Mental Health: Problems and Priorities in Low-Income Countries. Oxford University Press, 1995.

2 World Health Organization. World Health Report 2001. Mental Health: New Understanding, New Hope. WHO, 2001.

3 Lancet Global Mental Health Group. Scaling up services for mental disorders - a call for action. Lancet 2007; 370: 1241-52.

4 Patel V, Thornicroft G. Packages of care for mental, neurological, and substance use disorders in low- and middle-income countries: PLOS Medicine Series. PLOS Med 2009; 6: e1000160.

5 World Health Organization. Mental Health Gap Action Programme (mhGAP): Scaling up Care for Mental, Neurological and Substance Abuse Disorders. WHO, 2008.

6 Thornicroft G, Brohan E, Rose D, Sartorius N, Leese M. Global pattern of experienced and anticipated discrimination against people with schizophrenia: a cross-sectional survey. Lancet 2009; 373: 408-15.

7 Kleinman A. Global mental health: a failure of humanity. Lancet 2009; 374 603-4.

8 Patel V, Prince M. Global mental health: a new global health field comes of age. JAMA 2010; 303: 1976-7. 